\title{
Modell fuilr eine kooperative Schwangerenvorsorge von Ärztin und Hebamme
}

\author{
Christine Laß, Juliette Becker
}

Eine kompetente Begleitung und Beratung in der Schwangerschaft zählt zum originären Aufgabenfeld der Hebammen. Dennoch wird die Schwangerenvorsorge gemäß den Mutterschaftsrichtlinien meist mit ärztlicher Betreuung assoziiert. Unsere Autorinnen stellen ihr Kooperationsmodell vor, bei dem Hebamme und Gynäkologin schwangeren Frauen eine gemeinsame Vorsorge anbieten. Nachfolgend beschreiben sie, wie eine Zusammenarbeit der Professionen auf Augenhöhe gelingen kann und welche Vorteile sie für die Ratsuchenden bietet.

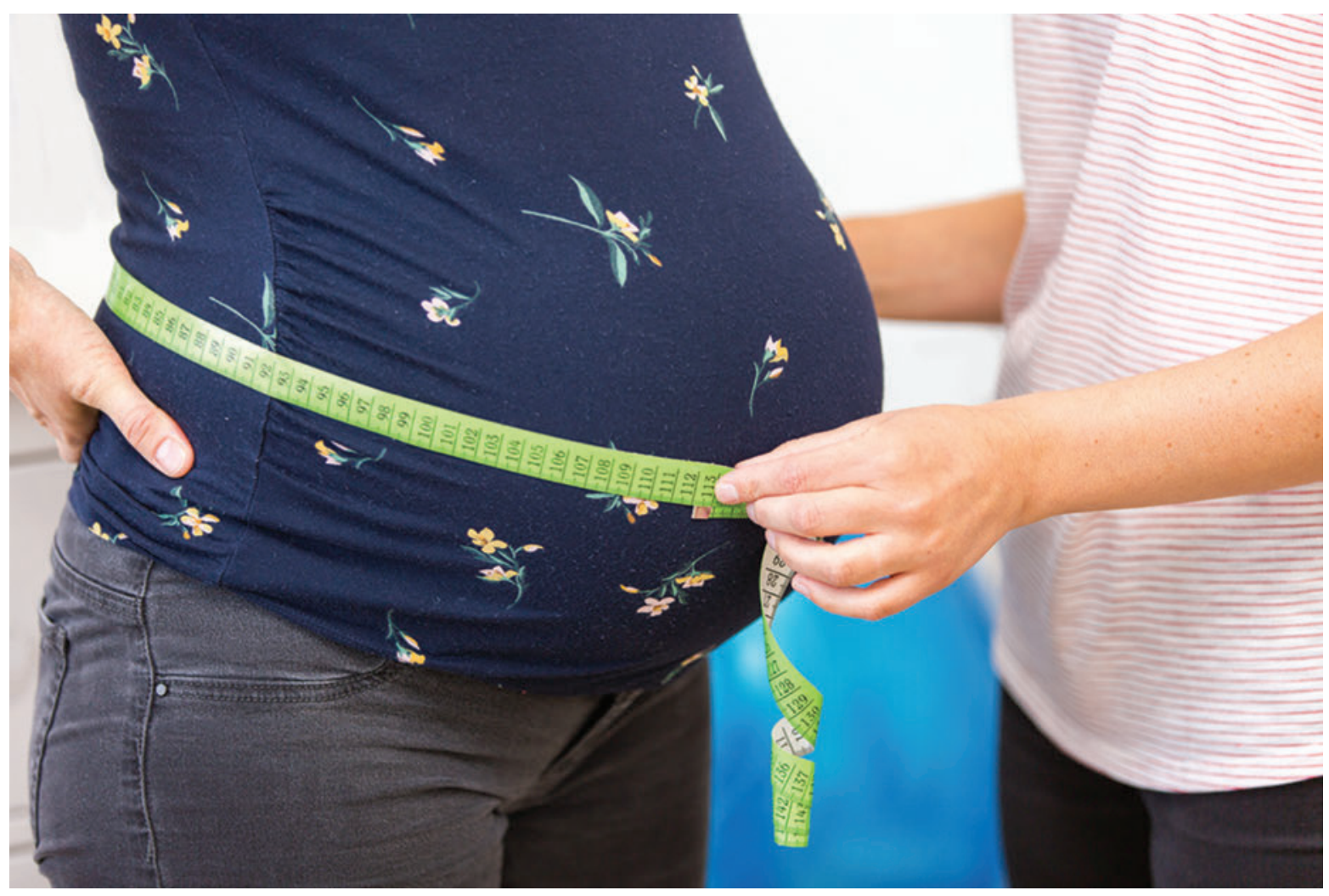

Abb. 1 Ob im vorgestellten Kooperationsmodell die Hebamme wie hier im Bild oder die Gynäkologin den Bauchumfang kontrolliert, hängt auch davon ab, in welcher SSW der Vorsorgetermin stattfindet. Die gelungene Zusammenarbeit basiert auf einer klar geregelten Aufgabenverteilung und Ablaufplanung. Zudem müssen die Partnerinnen in wichtigen Fragen der Schwangerenvorsorge übereinstimmen bzw. ihre Kompetenzen klar abgrenzen. (Quelle: Kirsten Oborny / Thieme Mediengruppe)

\section{Hintergrund}

Es gibt verschiedene Formen der kooperativen Betreuung von Schwangeren. Meist wird sie von niedergelassenen
Gynäkologinnen angeboten, wenn die Frauen zu den ersten Beratungen in der Praxis erscheinen. Aus dem Kolleginnenkreis sind uns sehr unterschiedliche 
Kooperationsmodelle bekannt. Manche Hebammen sind in der Praxis angestellt und übernehmen auch Aufgaben der Medizinischen Fachangestellten (MFAs). Andere arbeiten ungeregelt, je nach Bedarf, in einer gynäkologischen Praxis mit. Diese konzeptfreien Kooperationsmodelle spiegeln sowohl die Unklarheiten und Unsicherheiten in der Interpretation der Mutterschaftsrichtlinien als auch in der Abrechenbarkeit der Leistungen von Hebammen und Gynäkologinnen wider. Die berufspolitische Auseinandersetzung um das Thema ist bekannt und hat in den vergangenen Jahren zur Auflösung von Gyn-HebammenKooperationen geführt.

Im Folgenden möchten wir darstellen, mit welchem Konzept und nach welchen Regeln wir als Hebammen mit Gynäkologinnen die Schwangerenvorsorge gemeinsam anbieten. Es ist die Essenz aus unseren über Jahre gesammelten Erfahrungen.

\section{DIE AUTORINNEN}

\section{Die Praxis-Kooperation der Hebammen}

Beide Autorinnen kooperieren mit gynäkologischen Praxen:

Christine Laß arbeitet seit 15 Jahren in der Praxis von Julia Tilgner in Northeim (vormals bis 2013 Praxis Dr. med. Claudia Schumann [4]) und seit 2018 zudem in der gynäkologischen Praxis Dr. med. Kathrin Tepper-Wessels in Göttingen.

Juliette Becker lernte das Kooperationsmodell als Praktikantin vor der Hebammenausbildung kennen. Auch sie kooperiert aktuell mit zwei Gynäkologinnen - seit 2017 in der Praxis Dr. med. Mirjam Marwedel und Dr. med. Isabell Kampmann-Küster in Hann. Münden sowie seit 2013 in der Praxis Marc-Oliver Blaschke in Göttingen.

Die Entscheidung für eine Kooperation war für beide Hebammen eine bewusste Entscheidung. Sie ist nicht aus einer Not heraus entstanden, sondern aus der Überzeugung, dass dieses Modell der Schwangerenbetreuung auch für die Frauen das Beste ist. Mit dem Konzept werden Frauen erreicht, die sonst erst kurz vor der Geburt eine Hebamme kennenlernen, weil ihnen die Möglichkeit der Schwangerenvorsorge durch eine Hebamme vorher nicht bekannt war. Der erste Vorsorgetermin bei der Hebamme findet im vorgestellten Kooperationsmodell planmäßig in der 10.-12. SSW statt (vgl. \Tab. 1). Bis dahin ist die Schwangerschaft festgestellt und in der Sonografie bestätigt. Die Ärztin hat die Anamnese durchgeführt und die Schwangere zu Ernährung, Arbeit, Nikotinund Alkoholkonsum sowie Pränataldiagnostik beraten.

Der erste Besuch bei der Hebamme in der Praxis ist für viele Frauen etwas Besonderes, obwohl sie schon oft in dieser Praxis waren. Schließlich besiegelt er noch einmal die bestehende Schwangerschaft. Ihr
Bauch und somit ihr Kind wird ertastet. Sie hört die kindlichen Herztöne und lässt sich über das Leistungsspektrum der Hebammen informieren. Es ist viel Raum für Fragen, es werden Kurse und auch die Wahl der Geburtsklinik besprochen.

Zurzeit werden in den kooperierenden Praxen alle Graviden, die es wünschen, auch von den beiden Hebammen mitbetreut. Sind es sehr viele Schwangere, wird die Arbeitszeit in der Praxis entsprechend verlängert.

\section{Warum eine Kooperation sinnvoll ist}

Unseres Erachtens bietet die Kooperation Vorteile gegenüber dem alleinigen Angebot der Vorsorgeuntersuchungen in eigener Hebammenpraxis:

- Wir gehen in eine bestehende, laufende (Gynäkologinnen-)Praxis. Räume sowie Ausstattung sind vorhanden.

- Für die Terminplanung und damit für den reibungslosen Ablauf in der Praxis sind die MFAs zuständig.

- Bei Krankheit der Hebamme oder Urlaub der Praxis werden die Vorsorgen von der jeweils anderen Kooperationspartnerin durchgeführt. Die schwangere Frau bleibt in der Betreuung der Praxis.

- Bei in der Vorsorge erkannten Problemen, z. B. wenn die Schwangere von belastendem Stress mit dem Arbeitgeber erzählt, oder gar bei festgestellten Pathologien ist eine schnelle Hinzuziehung der Gynäkologin möglich. Die Gravide wird nicht zu einem anderen Arzt oder in die Klinik geschickt, was in einer Hebammenpraxis in einem solchen Fall notwendig wäre.

Diese Art des Vorgehens verschafft der Patientin, Ruhe, Sicherheit und Vertrauen. Sie führt auch bei der betreuenden Hebamme zu mehr Zufriedenheit mit der Arbeit. Die Frau wird von zwei Fachfrauen gesehen, Probleme werden zusammen besprochen und bestenfalls direkt gelöst. Auch das weitere Vorgehen wird besprochen und von beiden getragen. Die Verantwortung lastet nicht allein auf den Schultern der Hebamme oder der Gynäkologin. Den schwangeren Frauen gibt dies Sicherheit, wie sie auf Nachfrage bestätigen.

\section{Das sagen die Frauen}

Die Vorteile für die Graviden sollen hier beispielhaft anhand von Zitaten aufgezeigt werden:

„Hebamme oder Ärztin bei der Vorsorge? Ich hatte beides und fand es super. Und das alles in der gewohnten Praxis meiner Frauenärztin mit ihrem netten und kompetenten Team. In Kooperation mit einer Hebamme, die wie in meinem Fall miteinander gut kommunizieren, ist das 
Rundum sorglos-Paket komplett. Durch die Kooperation konnte ich meine Hebamme, welche mich im Wochenbett betreuen sollte, schon vorher gut kennenlernen und fühlte mich sicher und gut aufgehoben. Als meine Ärztin im Urlaub war, konnte ich zu meiner vertrauten Hebamme gehen und musste nicht zu einer fremden Ärztin. “

Frau M., betreut in der Praxis Dr. med. Kathrin Tepper-Wessels / Christine Laß in Göttingen

\begin{abstract}
„Meine Hebamme ist mit in meiner Frauenarztpraxis angesiedelt. Ich finde das sehr gut und unkompliziert. Schon früh in der Schwangerschaft konnte ich einen unverbindlichen Kennenlern-Termin mit der hausinternen Hebamme vereinbaren. Ganz unkompliziert und praktisch - falls ich mich nicht wohlgefühlt hätte, hätte ich mir auch außerhalb jemanden suchen können. Für mich war auch ein ausschlaggebender Punkt, dass die Hebamme meine Akte aus der Praxis kannte und direkt Vorsorge-Termine übernehmen konnte. Ich konnte während der normalen CTGs schon mal die eine oder andere Frage oder Unsicherheit besprechen. Dafür hätte ich vermutlich nicht extra einen Termin mit einer externen Hebamme gemacht oder jemanden angerufen. Auch von der Akupunktur bin ich ein Fan. Ich bin überzeugt, dass diese meine schnelle Geburt durchaus begünstigt hat. Das Kennenlernen vor der Geburt war super, da ich nach der Geburt direkt jemanden hatte, dem ich schon vertraute. So hatte ich keine Hemmungen, mich nach der Geburt als emotionales Hormonbündel zu präsentieren. Die Nachsorge war für mich Gold wert, ich habe mich auf jeden Termin gefreut und hoffe, dass wir weiter in Kontakt bleiben.
\end{abstract}

Frau T., betreut in Praxis Julia Tilgner/Christine Laß in Northeim

\section{Das sagt die kooperierende Gynäkologin}

Gynäkologin Julia Tilgner hat 2013 die Praxis von Dr. Claudia Schumann in Northeim übernommen, mit der Christine Laß bereits seit rund zehn Jahren kooperiert hatte. Die Kooperation führt sie seitdem fort und beschreibt deren Nutzen aus ärztlicher Sicht so:

„Die Vorteile sind die Zusammenarbeit in der Schwangerenvorsorge und der damit gegebene Austausch. Nachteile gibt es für mich als Gynäkologin nicht. Ich empfinde die Heb-Ärzte-Kooperation als absolute Bereicherung der Gyn-geburtshilflichen Arbeit.

Die Frauen erleben 2 Seiten unserer Arbeit, die sich gut ergänzen. Insbesondere weil viele Hebammen erst nach der Geburt zur Wöchnerin kommen, ist die Kontaktaufnahme und Betreuung in der Schwangerschaft sehr wichtig und hier sehr beliebt: Viele Fragen werden geklärt, für die sonst wenig Zeit ist, viele Zusatzangebote von Akupunktur über Taping werden genutzt. Die Frauen fühlen sich sicherer, vermeiden Krankenhausaufenthalte durch kontinuierliche Anleitung und gutes Körpergefühl.

Für mich stellt die Kooperation auch eine Arbeitsentlastung dar - weniger Kontakte, aber mit der Möglichkeit, Problemfälle zu besprechen - auch hier vermeiden wir oft unnötige Kontrollen im Krankenhaus, wenn wir uns absprechen und die Verantwortung splitten."

Julia Tilgner, niedergelassene Gynäkologin, Northeim

\section{Basis der Kooperation}

Die Kooperationspartnerinnen müssen gut gewählt sein. Für eine gute Zusammenarbeit braucht es neben Sympathie auch die Anerkennung des Könnens der jeweils anderen. In der Einstellung zu wichtigen Anteilen der Schwangerenvorsorge, wie etwa Verfahren der Pränataldiagnostik, Sonografie oder dem Einsatz des CTGs, muss in den meisten Punkten Konsens herrschen. In Fragen, die eine Sache der Abwägung sind, werden Vereinbarungen getroffen, z. B. dass sich die Hebamme bei strittigen Themen wie der Grippeimpfung aus der Beratung und Durchführung heraushält.

Sind diese Punkte für beide Parteien geklärt und zufriedenstellend geregelt, gilt es, den Rahmen der Zusammenarbeit festzulegen. Dies beinhaltet etwa:

- Tage und Stundenumfang

- Festlegung von Verantwortlichkeiten

- Aufgaben der Hebamme

- Abgabe an Praxis für Nutzung von Raum und Equipment

Dies wird im Kooperationsvertrag fixiert. Abb. 1 zeigt beispielhaft einen von einer Autorin genutzten Vertrag. Es kann ebenso die Vorlage des Hebammenverbandes genutzt werden.

\section{Wer macht was?}

Der Ablauf und die Aufgaben- und somit auch Terminverteilung müssen festgelegt werden. Dies dient den MFAs als Grundlage für eine sinnvolle Terminvergabe (bei anstehendem Sonografie-Termin bei der Ärztin).

Ein Ablaufplan bietet den Rahmen, in dem sich die Terminvergabe, Intervall und auch die Frage bewegen, wem der jeweilige Termin zugeordnet wird (Ärztin oder Hebamme). Ereignisse, die einen Extratermin notwendig machen, kommen auch ohne Ablaufplan vor. Sobald wie möglich wird wieder an dem Plan festgehalten. Tab. 1 zeigt beispielhaft einen Ablaufplan der Autorinnen: 
Der Ablaufplan, der den MFAs, der Ärztin sowie der Hebamme vorliegt, mindert das Risiko, dass etwas vergessen wird, z. B. eine Laboruntersuchung oder die Gabe von Anti D. Bei jedem Treffen wird kurz abgeglichen (Mutterpass, Praxiskarte und Ablaufplan), ob alle Untersuchungen zeitgerecht durchgeführt wurden.

Er bietet auch eine gute Basis für die Evaluation in der Praxis. Bei den Teamsitzungen und den Fallbesprechungen wird schnell deutlich, wo etwas versäumt wurde, fehlerhaft war und was verbessert werden kann.

Darüber hinaus macht jede Hebamme ihre eigene Evaluation für ihr internes Audit.

\section{Abrechnung}

Ein heikler Punkt bei der Kooperation ist die Abrechnung. Wer darf was abrechnen? Macht die Ärztin Verlust, wenn die Frauen zur Hebamme gehen? Entsprechend ist es oft die erste Frage, welchen Gewinn oder Nutzen die Praxis durch eine etwaige Kooperation hat.

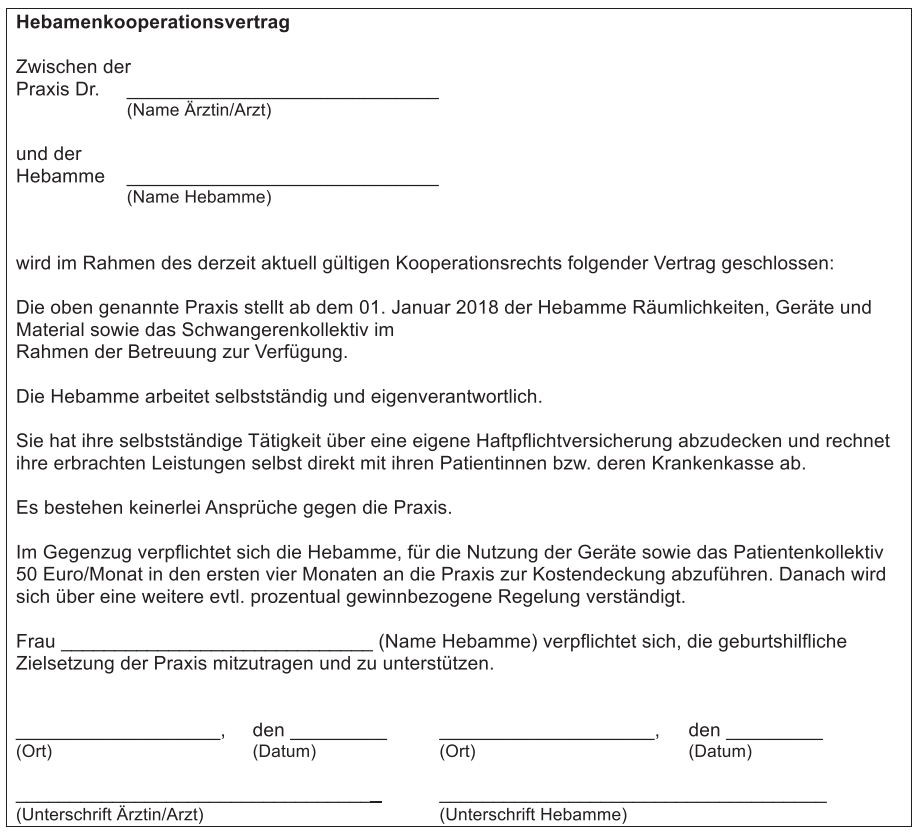

Abb. 2 Mustervereinbarung Zusammenarbeit Hebamme und Arzt / Ärztin (Quelle: eigene Darstellung)

- Tab. 1 Ablaufplan Schwangerenvorsorge Hebamme und Arzt / Ärztin im Sinne einer Praxiskooperation.

Bei jedem Termin / Kontakt: Frage nach subjektivem Befinden und nach Sorgen oder Beschwerden.

Bei Beschwerden: vaginale Untersuchung, pH-Bestimmung oder Cervixsonografie.

\begin{tabular}{|c|c|c|c|}
\hline SSW & $\begin{array}{l}\text { Untersuchungen in Anlehnung an } \\
\text { Mutterschaftsrichtlinien }\end{array}$ & Blutentnahmen / Labor & Wer? \\
\hline 05. -07 . & $\begin{array}{l}\text { " Feststellung der Schwangerschaft } \\
\text { " Bestimmung des voraussichtlichen } \\
\text { Geburtstermins } \\
\text { " Erhebung der Anamnese und Beratung } \\
\text { über die Ernährung, Veränderungen in } \\
\text { der Schwangerschaft, Beratungsstellen }\end{array}$ & $\begin{array}{l}\text { " Blutgruppenbestimmung } \\
\text { " Ak-Suchtest } \\
\text { " Röteln, HB, Lues, Chlamydien } \\
\text { " HIV (möglich) } \\
\text { " Toxoplasmose, Listerien, CMV und Parvovirus } \\
\text { auf Wunsch möglich }\end{array}$ & Arzt \\
\hline $08 .-12$. & $\begin{array}{l}\text { " Übergabe Mutterpass } \\
\text { " I Ultraschallscreening, evtl. } \\
\text { Cervixsonografie } \\
\text { " Ggf. NT-Messung, Harmony-Test } \\
\text { " Kontrolle der Vitalparameter der Frau } \\
\text { (Kontrolle: Gewicht, BMI, Urin, Blutdruck, } \\
\text { Ödeme, Varikosis) } \\
\text { " Ausführliches Beratungsgespräch über } \\
\text { Hebammenhilfe, Wochenbettbetreuung, } \\
\text { Hilfe bei Schwangerschafsbeschwerden, } \\
\text { Kurse }\end{array}$ & & $\begin{array}{l}\text { Arzt } \\
\text { Arzt } \\
\text { Arzt } \\
\text { Hebamme } \\
\text { Hebamme }\end{array}$ \\
\hline 16. -18. & $\begin{array}{l}\text { " Beratung/ Gespräch } \\
\text { " Kontrolle der Vitalparameter der Frau } \\
\text { " Kontrolle kindlicher Herztöne (mit Soni- } \\
\text { caid) und Fundusstand, Symphysen- } \\
\text { Fundusabstand, Bauchumfang }\end{array}$ & $\begin{array}{l}\text { " ggf. Rötelnkontrolle bei fehlendem } \\
\text { Immunschutz } \\
\text { " ggf. Toxoplasmosekontrolle } \\
\text { " ggf. TSH-Kontrolle }\end{array}$ & Hebamme \\
\hline 18. -22. & $\begin{array}{l}\text { " II Ultraschallscreening } \\
\text { " Herz- und Organultraschall, ggf. } \\
\text { Geschlechtsbestimmung } \\
\text { " Kontrolle der Vitalparameter der Frau }\end{array}$ & & Arzt \\
\hline
\end{tabular}




\begin{tabular}{|c|c|c|c|}
\hline \multirow[t]{2}{*}{ SSW } & $\begin{array}{l}\text { Untersuchungen in Anlehnung an } \\
\text { Mutterschaftsrichtlinien }\end{array}$ & Blutentnahmen / Labor & Wer? \\
\hline & $\begin{array}{l}\text { Kontrolle kindlicher Herztöne und Fun- } \\
\text { dusstand Symphysen-Fundusabstand, } \\
\text { Bauchumfang }\end{array}$ & & \\
\hline 24.-28. & $\begin{array}{l}\text { Kontrolle der Vitalparameter der Frau } \\
\text { " Kontrolle kindlicher Herztöne und Fun- } \\
\text { dusstand Symphysen-Fundusabstand, } \\
\text { Bauchumfang } \\
\text { - } \text { oraler Glukosetoleranztest }\end{array}$ & $\begin{array}{l}\text { " Hb-Kontrolle } \\
\text { " } 2 \text {. Ak.-Suchtest } \\
\text { " Blutzuckerbestimmung } \\
\text { " IGeL Toxoplasmosekontrolle }\end{array}$ & Hebamme \\
\hline 28.-32. & $\begin{array}{l}\text { " III Ultraschallscreening } \\
\text { " Kontrolle der Vitalparameter der Frau } \\
\text { " Kontrolle kindlicher Herztöne und Fun- } \\
\text { dusstand, Symphysen-Fundusabstand, } \\
\text { Bauchumfang }\end{array}$ & " Anti-D-Prophylaxe bei Rh-neg Frauen & $\begin{array}{l}\text { Arzt } \\
\text { Hebamme } \\
\text { Hebamme }\end{array}$ \\
\hline 32.-34. & $\begin{array}{l}\text { - Kontrolle der Vitalparameter der Frau } \\
\text { " Kontrolle kindlicher Herztöne (CTG) } \\
\text { " Kontrolle Fundusstand, Symphysen- } \\
\text { Fundusabstand, Bauchumfang } \\
\text { - Bescheinigung über Mutterschutz }\end{array}$ & - HBs-Antigen (Screening auf Hepatitis B) & Arzt/Hebamme \\
\hline 34.-36. & $\begin{array}{l}\text { - Kontrolle der Vitalparameter der Frau } \\
\text { - Kontrolle kindlicher Herztöne (CTG) } \\
\text { - Kontrolle Fundusstand, Symphysen- } \\
\text { Fundusabstand, Bauchumfang } \\
\text { - } \text { ggf. vaginale Untersuchung }\end{array}$ & - ggf. Abstrich auf Beta-Streptokokken (IGeL) & Hebamme \\
\hline 36.-38. & $\begin{array}{l}\text { " Kontrolle der Vitalparameter der Frau } \\
\text { - Kontrolle kindlicher Herztöne (CTG) } \\
\text { - } \text { Fundusstand, Symphysen-Fundusab- } \\
\text { stand, Bauchumfang } \\
\text { - } \text { Abschluss Arztgespräch }\end{array}$ & & Arzt/Hebamme \\
\hline 38. -40. & $\begin{array}{l}\text { - Kontrolle der Vitalparameter der Frau } \\
\text { - } \text { Kontrolle kindlicher Herztöne (CTG) } \\
\text { - } \text { Fundusstand, Symphysen-Fundusab- } \\
\text { stand, Bauchumfang } \\
\text { - } \text { Abschlussgespräch Hebamme }\end{array}$ & & Hebamme \\
\hline \multicolumn{4}{|c|}{$\begin{array}{l}\text { Dieser Plan ist individuell für Praxen anpassbar. Die hier angeführten Termine für die Abschlussgespräche sind keine fixen Termine. Dies } \\
\text { bedeutet, dass die Betreuung auch über den errechneten Entbindungstermin hinaus fortgeführt werden kann. Einfluss auf das weitere } \\
\text { Procedere haben der Verlauf der Schwangerschaft, schwangerschaftsbedingte Erkrankungen oder auch Vorerkrankungen der Frau } \\
\text { sowie die zeitliche Erreichbarkeit der Praxis (Wochenende, Feiertage, Praxisurlaub). }\end{array}$} \\
\hline
\end{tabular}

Die Angst, dass es für die Praxis ein Verlustgeschäft wird, ist der Hauptgrund, warum viele Gynäkologinnen die Kooperation ablehnen, oft noch nicht einmal darüber nachdenken. Die Angst vor einem Regress steht sofort im Raum. Diese Bedenken spiegeln die Unklarheiten bzw. Unsicherheiten bei der Abrechnung wider (vgl. oben). Sind die Abrechnungsfragen geklärt, entspannt sich die Situation und die Vorteile einer Kooperation treten in den Vordergrund.
Im Folgenden stellen wir die Möglichkeiten der Abrechnung dar. Dies kann auch als Argumentationshilfe bei einem ersten Kooperationsgespräch genutzt werden.

Der Grundsatz lautet: Jede Kooperationspartnerin rechnet das ab, was sie leistet. Wird ein suspektes CTG von der Hebamme geschrieben und von der Ärztin mit beurteilt, rechnen es beide ab (Ziffer 0600). 


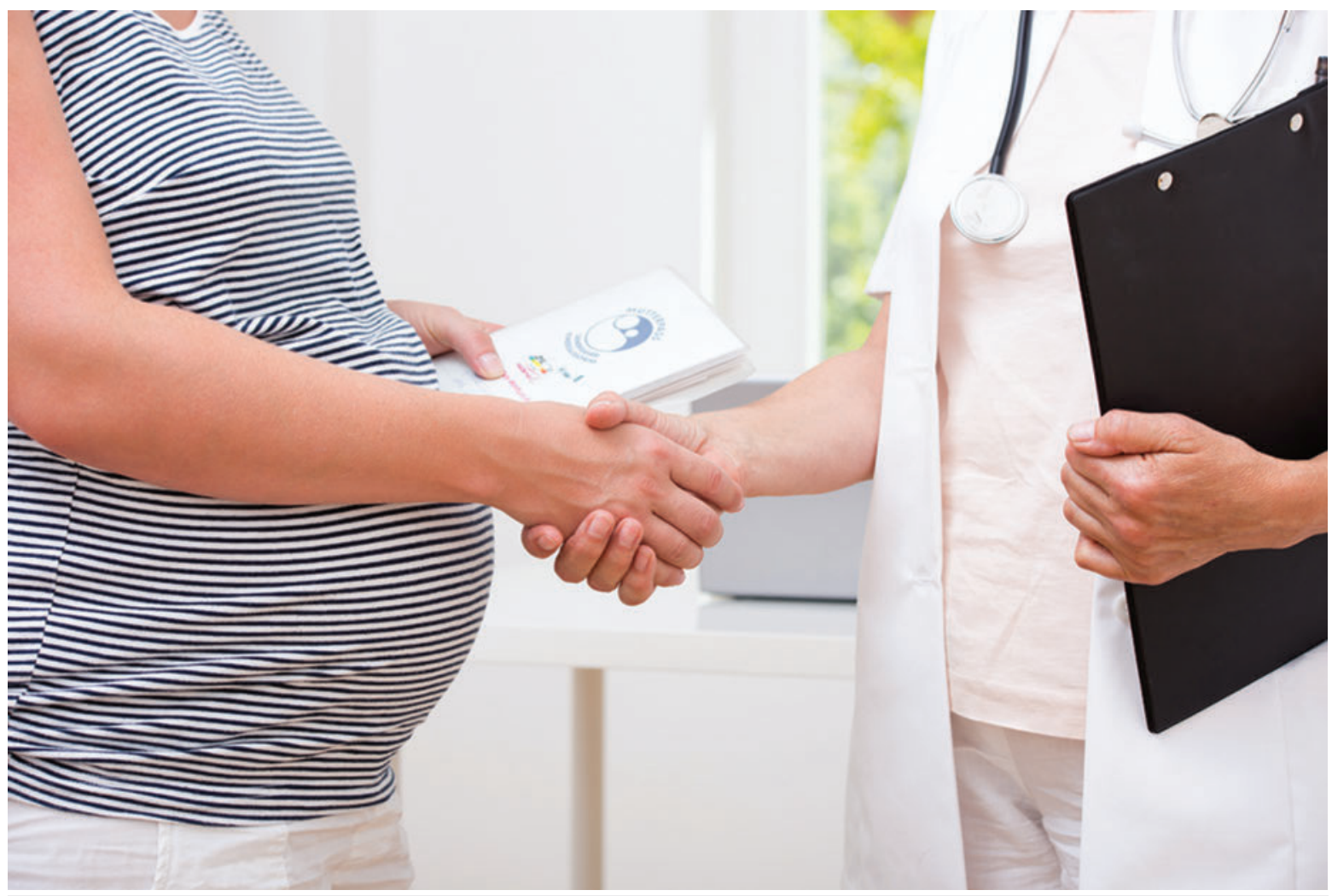

Abb. 3 Das abschließende Arztgespräch ist im vorgestellten Ablaufplan für die 36.-38. SSW vorgesehen, das Abschlussgespräch mit der Hebamme für die 38.-40. KW. (Quelle: drubig-photo - stock.adobe.com)

Ein weiteres Beispiel: Eine schwangere Frau war zur Vorsorge bei der Ärztin. Dort wurde eine beginnende Symphysenlockerung diagnostiziert. Die Ärztin bietet ihr an, sich am folgenden Tag bei der Hebamme mit einem Kinesiotape behandeln zu lassen. Da das Hilfsangebot niedrigschwellig und schnell umzusetzen ist, wird es von den Frauen sehr gern angenommen. Die Hebamme kann in diesem Fall die Ziffer 0500 Hilfe bei Schwangerschaftsbeschwerden abrechnen. Der Termin hierfür wird von den MFAs vergeben.

Die Ziffern 0200/0230 sollten nur dann abgerechnet werden, wenn die Hebamme auch die Wochenbettbetreuung übernimmt.

\section{FAZIT}

\section{Vorteile der Kooperation von \\ Hebamme und Ärztin}

Viele positive Aspekte der Kooperationen wurden von uns beschrieben. Das Modell weist klare Vorteile für die Hebamme auf. Wir sparen Zeit, Praxisräume und Ausstattung können mitgenutzt werden, die Terminvergabe geschieht durch die MFAs, das QM der Schwangerenvorsorge wird gemeinsam mit der Praxis durchgeführt.

Auch die Gynäkologin profitiert von der Kooperation, u. a. durch eine Arbeitsentlastung (weniger Kontakte) und die Möglichkeit, Problemfälle direkt
-Tab.2 Abrechnungsfähige Ziffern in Rahmen der Schwangerschaftsvorsorge

\begin{tabular}{|c|c|c|}
\hline Nummer 0300 & $\begin{array}{l}\text { Vorsorgeuntersuchung der } \\
\text { Schwangeren }\end{array}$ & 30,92 Euro \\
\hline Nummer 2500 & Entnahme von Körpermaterial & 7,87 Euro \\
\hline Nummer 0500 & $\begin{array}{l}\text { Hilfeleistung bei Schwangerschafts- } \\
\text { beschwerden oder Wehen }\end{array}$ & 20,70 Euro \\
\hline Nummer 0600 & $\begin{array}{l}\text { Kardiotokographische } \\
\text { Überwachung }\end{array}$ & 8,85 Euro \\
\hline Nummer 0100 & $\begin{array}{l}\text { Beratung der Schwangeren auch } \\
\text { mit Kommunikationsmedium }\end{array}$ & 8,00 Euro \\
\hline Nummer 0400 & GDM Screening & 9,83 Euro \\
\hline Nummer 0200 & $\begin{array}{l}\text { Individuelle Basisdatenerhebung } \\
\text { und Leistungsauskunft }\end{array}$ & 32,02 Euro \\
\hline Nummer 0230 & $\begin{array}{l}\text { Individuelles Vorgespräch über Fra- } \\
\text { gen der Schwangerschaft und } \\
\text { Geburt }\end{array}$ & 44,60 Euro \\
\hline Quelle: Hebam & iilfevertrag 2018 [3] & \\
\hline
\end{tabular}

mit der Hebamme zu besprechen (Vermeiden unnötiger Krankenhauskontrollen).

Im Fokus der gemeinsamen Betreuung steht die schwangere Frau: Die frauenorientierte 
Schwangerenberatung ist förderlich für das Selbstwertgefühl der Frau und ihr Vertrauen in die Funktion und Kraft des eigenen Körpers. Ihr Kohärenzgefühl wird gestärkt, im Sinne von Antonovsky [1]. Die Schwangere erfährt, was sich in der Schwangerschaft verändert, warum sich etwas ändert und wie sie damit umgehen kann. Dies ist auch eine gute Vorbereitung auf die anstehende Geburt, weil Ungewissheit und Ängste vor Schmerzen und Kontrollverlust gemindert werden und das Vertrauen in die eigene Selbstwirksamkeit gestärkt wird.

In diesem Sinne werden auch Themen wie „Wunschkaiserschnitt“, Geburtsvorbereitungskurse, Wochenbettbetreuung oder der Einsatz von Familienhebammen frühzeitig besprochen. Beide würden wir unseren Kolleginnen vorbehaltlos zu diesem Kooperationsmodell raten.

\section{Autorinnen / Autoren}

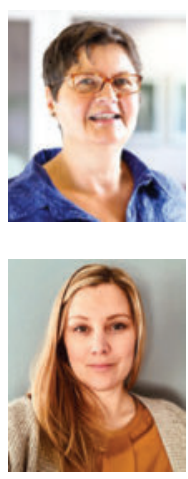

Christine Laß, MA Soziologie / Pädagogik, ist Lehrerin für Hebammenwesen, freiberufliche Hebamme in Göttingen und Northeim

Juliette Becker ist freiberufliche Hebamme in Göttingen

\section{Korrespondenzadresse}

Christine Laß

E-Mail: c.lass@gmx.net

\section{Literatur}

[1] Antonovsky A. Salutogenese. Zur Entmystifizierung der Gesundheit. Tübingen; DGVT-Verlag 1997

[2] Gemeinsamer Bundesausschuss. Mutterschaftsrichtlinien. 2016; www.g-ba.de/informationen/richtlinien/19/

[3] GKV-Spitzenverband. Hebammenhilfevertrag. 2018; www. gkv-spitzenverband.de/krankenversicherung/ambulante_leis tungen/hebammen/hebammenhilfevertrag/hebammenhilfe vertrag.jsp

[4] Laß C, Schumann C. Warum eine Kooperation von Ärztin und Hebamme auf Augenhöhe sinnvoll und machbar ist. Die Hebamme 2016; 29: 204-206

\section{Bibliografie}

DOI https://doi.org/10.1055/a-0861-0479

Die Hebamme 2019; 32: 44-50

(c) Georg Thieme Verlag KG Stuttgart · New York ISSN 0932-8122 